\title{
DIE ZUKUNFT DER FAMILIENBETRIEBE IN EUROPA
}

(Vortrag gehalten am 21. September 1961 in der Finnischen Agrikulturwissenschaftlichen Gesellschaft)

Paul Rintelen

Institut für Wirtschaftslehre des Landbaues der Technischen Hochschule München,
Freising-Weihenstephan

Die beiden Welten, die sich heute gegenüberstehen, haben in Bezug auf die Agrarverfassung eine grundlegend verschiedenartige Konzeption. In den zum Sowjetblock gehörenden Ländern finden wir die Sowchose und die Kolchose, also die Grossbetriebsform, mit dem nach Urteil Sachverständiger zu erkennenden Ziel, das Kollektiveigentum auf die Stufe des Volkseigentums allmählich zu bringen. Im westlichen Einflussbereich glaubt man den Boden am besten in der Hand eines freien Bauerntums, das seinem Wesen nach am günstigsten in die gesamte Wirtschafts- und Sozialordnung zu passen scheint. Hier die Vorstellung von dem Massenmenschen, der sich leicht lenken und leiten lässt, dort von dem Menschen als freien Individualisten, der sich aber gebunden fühlt durch Moral, Sitte und Recht, den 3 Grundpfeilern, auf denen unser Gebäude der Sozialordnung steht.

Auf jeden Fall besteht in den westlichen Ländern der Wille, die bäuerliche Familienwirtschaft zu erhalten. Das ist auch deutlich beim Abschluss des EWGVertrages in Rom 1957 zum Ausdruck gekommen, wo sich die beteiligten 6 Länder Italien, Frankreich, Belgien, Luxemburg, Niederlande und Westdeutschland dahingehend festgelegt haben, dass die agrarpolitischen Massnahmen so zu handhaben sind, dass die Existenz der bäuerlichen Familienwirtschaft gesichert bleibt. Demzufolge sind auch im EWG-Vertrag entsprechende zusätzliche Formulierungen aufgenommen worden, die den Fortbestand der Familienwirtschaft garantieren sollen. Da sich in der Zwischenzeit die EFTA-Länder anschicken, mit der EWG zu einem grosseuropäischen Wirtschaftsblock zusammen zu wachsen und da in den EFTALändern die Landwirtschaftspolitik ebenfalls auf die Erhaltung des Familienbetriebes ausgerichtet ist, so dürfte kein Zweifel bestehen, dass auch in einem europäischen Wirtschaftsblock eine Agrarpolitik betrieben wird, die den Familienbetrieb zu erhalten bestrebt ist.

Bevor nun auf das eigentliche Problem, nämlich die Zukunft des Familienbetriebes in Europa, eingegangen wird, ist es notwendig, zunächst eine Definition 
darüber zu geben, was unter einem Familienbetrieb zu verstehen ist. Agrarsoziologisch werden bekanntlich nach der Arbeitsverfassung unterschieden:

1. der Nebenerwerbsbetrieb,

2. der Familienbetrieb,

3. der Gesindebetrieb und

4. der Lohnarbeitsbetrieb.

Die Grenzen zwischen den genannten sind selbstverständlich fliessend.

Betriebswirtschaftlich werden die Betriebe meistens nach der Grösse aufgegliedert. Es sei an dieser Stelle schon betont, dass der Familienbetrieb an keine bestimmte Betriebsgrösse gebunden ist. Es gibt Familienbetriebe mit sehr geringen landwirtschaftlichen Flächen, wo dann aber häufig Spezialkulturen, die einen sehr hohen Rohertrag pro Flächeneinheit abwerfen, angebaut werden. Auf der anderen Seite gibt es aber auch Familienbetriebe mit recht erheblichen Flächenarealen. Es ist daher falsch zu glauben, dass der Familienbetrieb nur in einer Grössenordnung zwischen vielleicht 5 und 15 ha gegeben sei, wie das vielfach zu hören ist. Gewiss liegt die Masse der Familienbetriebe in dieser Grössenordnung, aber es braucht nicht so zu sein, es gibt auch Familienbetriebe mit 100 ha landwirtschaftlicher Nutzfläche, wenn sie auch zahlenmässig sehr gering sind.

Entsprechend den Wesensmerkmalen des Familienbetriebes kann dieser wie folgt definiert werden: Ein Familienbetrieb ist eine landwirtschaftliche Unternehmung, die dadurch gekennzeichnet ist, dass sie eine Grösse besitzt, die einer Familie ein mit anderen Berufsgruppen vergleichbares Einkommen bei ordnungsgemässer Bewirtschaftung abwirft, fast ausschliesslich mit familieneigenen Arbeitskräften bearbeitet wird und bei der der Betriebsleiter eigenverantwortlich wirtschaftet, eigene Verfügungsgewalt über die Produktionsfaktoren hat sowie das Risiko des Wirtschaftens trägt.

Das bisher Gesagte lässt bereits erkennen, dass die Erhaltung des Familienbetriebes zwar das Ziel der Politiker des Westens ist und vermutlich bleiben wird. Jedoch war bisher die Vorstellung von dem, was ein Familienbetrieb eigentlich ist, recht verschwommen. Insbesondere verband man gern diesen Begriff mit der Betriebsgrösse. Hier aber vollziehen sich im Augenblick, im Zeitalter der Hochkonjunktur, in der Bundesrepublik Westdeutschland, aber auch in anderen europäischen Ländern grosse Wandlungen, die in den nächsten beiden Jahrzehnten besonders deutlich zutage treten werden. Zur Zeit ist es z.B. in den wenig begünstigten Zonen der Bundesrepublik so, dass die landwirtschaftlichen Grenzbetriebe häufig nur noch von der alten Generation bewirtschaftet werden, die junge Generation sich aber anderen Berufen zugewandt hat, wo das Geld schneller und leichter zu verdienen ist als auf der kargen heimischen Scholle. Über 100000 dieser kleinbäuerlichen Familienbetriebe sind in Deutschland in den letzten 10 Jahren aufgelöst worden. Bei Fortbestehen der allgemeinen Wirtschaftslage ist mit Bestimmtheit damit zu rechnen, dass einige 100000 weiterer Betriebe der Auflösung verfallen werden.

Der tiefere Grund für diesen Vorgang ist darin zu erblicken, dass bei gleichen oder gar fallenden Agrarproduktenpreisen aber steigendem Einkommen in den vergleichbaren Berufsgruppen die Mindestbetriebsgrösse nicht mehr ausreicht, der 
bäuerlichen Familie im Kleinbetrieb ein der volkswirtschaftlichen Entwicklungsstufe entsprechendes Einkommen zu gewährleisten. In einer solchen Entwicklungsphase muss sich zwangsläufig der Zug zur grösseren Betriebseinheit für den Familienbetrieb entwickeln. Tatsächlich zeigen auch die Statistiken über die Zahl der Betriebe in den einzelnen Betriebsgrössenklassen für die meisten europäischen Länder einen Rückgang der Kleinst- und Kleinbetriebe und eine Zunahme bei den Mittelbetrieben und den grösseren Betrieben, nicht dagegen bei den ausgesprochenen Grossbetrieben. Je höher die allgemeine Wirtschaftshausse eines Landes ist, umso stärker kommt diese Entwicklung zum Ausdruck.

Die gleichen Kräfte, die die Zahl der reinen Familienbetriebe in den unteren Betriebsgrössenklassen vermindern, erhöhen ihre Zahl in den höheren Betriebsgrössenklassen. Die Gesindebetriebe nämlich gehen an Zahl stark zurück und werden zu reinen Familienbetrieben. Die ledigen Arbeitskräfte werden vom Sog zum industriellen Arbeitsplatz besonders stark angezogen. Der Gesindebetrieb hat seit eh und je den Überschuss an Arbeitskräften einer Volkswirtschaft aufgenommen. Sobald aber auf dem Arbeitsmarkt ein Arbeitskräftemangel auftritt, werden die Gesindebetriebe zuerst ihrer Fremdarbeitskräfte beraubt. Es folgen dann die Söhne und Töchter der Kleinbauern. In dieser Situation stehen heute die landwirtschaftlichen Betriebe in den hochindustrialisierten Ländern des westlichen Kulturkreises.

Sekundär ist an diesem Entwicklungsprozess die Mechanisierung beteiligt, die den Vorgang zudem beschleunigen hilft. Der Kleinbauer, der die Arbeiten bisher mit Kuh-, Ochsen- oder Pferdegespann ausführte, merkt beim Übergang zum 15-20 PS Schlepper bald, dass seine Arbeitskraft nicht mehr ausgelastet ist. Er wird zunächst zu spezifisch intensiveren Betriebszweigen übergehen und darüber hinaus versuchen, Land von sich auflösenden Betrieben hinzu zu kaufen oder zuzupachten. Alsdann wird ihm klar, dass seine Arbeitsmacht sich weiter verstärkt, wenn er vom Kleinschlepper zur Schleppergrösse zwischen 35 und 60 PS übergeht. Damit vollzieht sich die Entwicklung zur weiteren Betriebsvergrösserung. Die Situation ist also derart, dass manche nach heutiger allgemeiner Wirtschaftslage zu kleine Familienbetriebe und auch Nebenerwerbswirtschaften die Landwirtschaft ganz oder teilweise aufgeben und dass das freiwerdende Land zur Betriebsaufstockung in anderen Betrieben verwandt wird, die damit zu lebensfähigen Familienbetrieben im Sinne der vorher gegebenen Definition werden.

Die Gesindebetriebe, die im Laufe des letzten Jahrzehnts in Westdeutschland zu reinen Familienbetrieben wurden, haben zunächst häufig unter zu starker Arbeitsbelastung der Familien-Arbeitskräfte gelitten und bei nicht wenigen ist es heute noch so. Zunächst hat man versucht durch erhöhte Arbeitsanstrengung der Familien-Arbeitskräfte den Betrieb aufrecht zu erhalten, nachdem Knecht und Magd abgewandert waren. Ohne Rücksicht auf eine mit der Mechanisierung notwendig werdende Betriebsumstellung und Betriebsvereinfachung wurden sodann Maschinen gekauft, die die bisher von Hand durchgeführten Arbeiten erledigen sollten. Die Maschinenkosten stiegen dabei nicht selten über die eingesparten Löhne hinaus und das Wirtschaftsergebnis wurde unbefriedigend. Diese Betriebe, die früher die Aufgabe hatten Arbeit und Brot zu liefern, stehen nunmehr vor der 
Aufgabe, mit möglichst wenig Arbeitskräften Nahrungsmittel zu produzieren. Folgerichtig müssen diese Betriebe heute auf die Betriebszweige ausgerichtet werden, die sich besonders gut mechanisieren lassen. Leitmotiv der Betriebsgestaltung war es in den Betrieben bisher im Sinne der Brinkmann'schen Theorie der Betriebsmittelgemeinschaft, einen besonders guten Arbeitsausgleich für Arbeitskräfte und Arbeitspferde zu finden. Bei beiden sind die Festkosten sehr hoch, die variablen Kosten gering. Die Situation in dieser Beziehung ändert sich mit dem Übergang zu Schlepper und Arbeitsmaschine.

Die Kosten der Mechanisierung sind bei mittel- bis grossbäuerlichen Betrieben durchaus tragbar, wenn Betriebsorganisation und Mechanisierung aufeinander abgestimmt sind. Das ist heute in der Mehrzahl der Betriebe durchaus noch nicht der Fall, wohl aber in den Pionierbetrieben. Die breite Masse wird diesen aber nachkommen.

Bleibt noch die Frage zu behandeln, ob nicht der Grossbetrieb gegenüber dem Familienbetrieb, besonders im Zeitalter der Mechanisierung und Automatisation, wirtschaftlich so im Vorteil liegt, dass er den Familienbetrieb verdrängen wird. Wenn das Herz auch für den Familienbetrieb schlägt, werden nicht die technische Fortentwicklung und andere wirtschaftliche Momente diesen doch zunichte machen? Es ist bekanntlich in der westlichen Welt so, dass die wirtschaftlichen Kräfte durchweg stärker sind als die politischen. Hier scheint es sinnvoll zu sein, die Vorund Nachteile, die Familien- und Grossbetriebe haben, gegeneinander abzuwägen, um so zu einer Urteilsbildung zu kommen.

1. Die Arbeit im landwirtschaftlichen Betriebe ist weitgehend an die Natur gebunden. Jahreszeit und Witterung bestimmen, welche Arbeiten ausgeführt werden müssen bzw. können. Auch bei in bezug auf die Arbeitsverteilung bester Betriebsorganisation lässt es sich nicht vermeiden, dass die Witterung im Arbeitsablauf Spitzen und Täler bedingt. Die Spitzen in Lohnarbeitsbetrieben zu bewältigen, bedeutet die Zahlung von tarifmässig festgelegten Überstunden oder mitunter auch von Sonntagsstunden. Ein Ausgleich mit Arbeitstälern ist nicht möglich. Die dort entstehenden Stunden müssen ebenfalls gezahlt werden. Die Arbeitskräfte werden mit häufig unwirtschaftlichen Arbeiten beschäftigt. — Im Familienbetrieb werden die Arbeitsspitzen leicht durch Überstunden bewältigt, die ja nicht besonders zu zahlen sind. Dafür kann die Familien-Arbeitskraft während der Arbeitstäler dem eigenen Hobby nachgehen. Auch besteht die Möglichkeit, zwischen häuslicher und aussenbetrieblicher Arbeit einen Austausch jederzeit vorzunehmen. Zur Zeit der Arbeitsspitze hilft die Bäuerin dem Bauern auf dem Felde, zur Zeit des Arbeitstales übernimmt der Bauer Arbeiten, die sonst der Bäuerin obliegen. In Bezug auf den für den Wirtschaftserfolg so wichtigen Arbeitsausgleich liegt der Familienbetrieb auf jeden Fall eindeutig im Vorteil, er vermag Arbeitsbedarf und Arbeitsmacht kurzfristig leichter auszugleichen.

2. Im landwirtschaftlichen Betrieb sind tagtäglich unternehmerische Entscheidungen zu treffen und danach ist zu handeln und zu arbeiten. Da im Familienbetrieb der Bauer gleichzeitig Unternehmer und Ausführender ist, treten keine Schwierigkeiten auf. Das ist aber im Grossbetrieb anders. Der Betriebsleiter kann nicht gleichzeitig an den verschiedenen Arbeitsplätzen des Betriebes sein, er muss 
schon einen Teil der unternehmerischen Entscheidungen an Untergebene delegieren. Besonders schwierig ist die Situation in staatlichen Grossbetrieben, wo der Betriebsleiter unternehmerische Entscheidungen nicht selten von übergeordneten Dienststellen einholen muss. Bis diese Entscheidungen eingetroffen sind, vergeht oft eine lange Zeite und der erhoffte Gewinn ist längst dahin.

3. Die Einzelarbeiten im landwirtschaftlichen Betriebe setzen sich zusammen aus der Rüstzeit, der Wegezeit und der eigentlichen Arbeitszeit am Beschäftigungsplatz. Bis zu einer gewissen Grösse der Viehherde oder des Feldstückes nehmen Rüst- und Wegezeit relativ ab, so dass von der gesamten aufgewandten Zeit für einen Arbeitsvorgang ein grösserer Anteil auf die eigentliche Arbeit entfällt, wodurch die Leistung ansteigt. Der so entstehende Leerlauf an menschlicher Arbeitskraft und an Maschinenstunden schlägt aber nur in den kleinen Familienbetrieben zu Buche, kaum in den grösseren. Bei letzteren insbesondere dann nicht, wenn diese in der Organisation vereinfacht sind, so dass die verbliebenen Betriebszweige einen genügenden Umfang einnehmen. Zudem dürfte die im Familienbetrieb steckende Privatinitiative und damit verbunden ein erhöhter Fleiss eventuelle Nachteile des Familienbetriebes gegenüber dem Grossbetrieb sicherlich ausgleichen, wenn nicht gar überkompensieren. - Werden die Betriebe zu gross, so nehmen die Wegezeiten wieder einen zu grossen Anteil von der Arbeitszeit in Anspruch. Das gilt besonders, wenn die Rindviehhaltung einen zu grossen Umfang einnimmt. Werden etwa 500 Kühe in einem Stall vereint, so sind bei Grünfütterung täglich etwa 30 to Futter und 20 to Stalldung zu transportieren. Wird das Futter nur auf den Stall-nahen Feldern produziert und der Stalldung auch dorthin gebracht, so leidet die symbiotische Wirkung zwischen Viehhaltung und Ackerbau. Das schlägt besonders stark in den hackfruchtstarken Betrieben zu Buche. Hier wird die Bodennutzungsgemeinschaft und die Verwertungsmeinschaft empfindlich gestört.

4. Zweifellos ist auch der Familienbetrieb bei der Produktion tierischer Nahrungsmittel überlegen. Bauer und Bäuerin gehen mit den gewonnenen Futtermitteln weit haushälterischer um, als das der Fremdarbeiter im Grossbetrieb kann. Eigene Untersuchungen in dieser Richtung haben gezeigt, dass bei gleichen Futtererträgen und gleicher tierischer Leistung der Grossbetrieb etwa $20 \%$ Futter mehr verbraucht als der Betrieb, in dem die Familien-Arbeitskräfte Fütterung und Pflege der Tiere selbst übernehmen. Die Situation kann bei den betriebsunabhängigen Viehhaltungszweigen, besonders bei der Geflügelhaltung, anders liegen. Aber auch hier ist zu bedenken, dass das Risiko bei zu grossen Beständen erheblich anwächst.

5. Auch die früher dem Grossbetrieb oft nachgesagte Besserstellung beim Einsatz von Maschinen trifft heute höchstens noch in bescheidenem Ausmasse zu. Der überbetriebliche Maschineneinsatz, die Betriebsvereinfachung und das richtige Abstimmen zwischen Betriebsorganisation und Technik lassen in vielen Fällen, besonders bei den grossbäuerlichen Familienbetrieben, diese durchaus konkurrenzfähig erscheinen.

6. Es wird weiter darauf hingewiesen, dass der Grossbetrieb in der Lage sei, die verschiedensten Spezialisten einzusetzen: den Melker, den Schweinemeister, den Schlepperfahrer usw. und er sei dadurch im Vorteil. Dies trifft zweifellos zu, soweit 
man den vielseitigen Familienbetrieb alter Prägung zum Vergleich heranzieht. In ihm kann der Bauer unmöglich auf allen Gebieten Spezialkenntnisse haben. Der Zug zur Betriebsvereinfachung und Spezialisierung lässt den Bauern aber selbst zum Spezialisten werden, insonderheit dann, wenn er eine entsprechende Ausbildung mitbekommen hat.

7. Letztlich sei noch erwähnt, dass das Tragen des Risikos im Familienbetrieb leichter möglich ist als im Grossbetrieb. Schlechte Zeiten können durch Einschränkung bei den persönlichen Ausgaben aufgefangen werden, das ist im Grossbetrieb, der Löhne zu zahlen hat, nicht möglich.

Alles in allem will es mir scheinen, dass beim Abwägen der Vor- und Nachteile von Grossbetrieb und Familienwirtschaft das Zünglein an der Waage entschieden zu Gunsten des Familienbetriebes ausschlägt, sicherlich dann, wenn dieser eine genügende Grösse hat und dementsprechend die Produktionsfaktoren Boden, Arbeit und Kapital zweckentsprechend aufeinander abgestimmt sind und wenn der Betriebsleiter eine gute fachliche Ausbildung erfahren hat.

Im westlichen Kulturkreis sind die Zukunftsaussichten für die Familienwirtschaft durchaus positiv zu beurteilen. Die Agrarpolitik und auch die allgemeine Politik ist in fast allen Ländern der westlichen Welt auf die Erhaltung eines gesunden Bauerntums eingestellt, das als wesentliche Stütze eines allgemein angestrebten Mittelstandes angesehen wird. Auch die ökonomischen Erwägungen, die wir über Grossbetrieb und Familienbetrieb anstellten, sprechen eindeutig zu Gunsten des Familienbetriebes. Nur muss man sich klar darüber sein, dass die allgemeine volkswirtschaftliche Entwicklung und die Verlagerung von der Handarbeit zur Maschinenarbeit in der Landwirtschaft eine Wandlung in der Betriebsgrössenstruktur nach sich zieht bzw. ziehen muss.

Wenn einleitend als Wesensmerkmal des Familienbetriebes herausgestellt wurde, dass dieser eine Grösse haben müsse, die der bäuerlichen Familie ein Einkommen liefert, welches mit anderen Berufsgruppen vergleichbar ist, so leuchtet ein, dass mit einer Produktivitätssteigerung in der übrigen Wirtschaft die Produktivität auch in der Landwirtschaft Schritt halten muss.

Eine Steigerung des Realeinkommens ist im grossen gesehen nur möglich, wenn der einzelnen Arbeitskraft mehr Kapital zugeordnet wird. In der Industrie bedeutet das im allgemeinen eine Vermehrung des Maschinenkapitals je Arbeitskraft. Die Maschinen werden komplizierter und teurer. In der Landwirtschaft aber gehört es zur Steigerung der Produktivität, dass je Arbeitskraft mehr Boden-, Vieh- und Maschinenkapital zur Verfügung steht. Zur Änderung der Produktivität in der Landwirtschaft ist es also im grossen gesehen notwendig, dass sich das Verhältnis zwischen Bodenfläche und Arbeitskraft wandelt derart, dass je Arbeitskraft mehr Boden vorhanden ist.

Hieraus resultiert, dass in Volkswirtschaften mit stark steigendem Wohlstand die Landwirtschaft nur Schritt halten kann, wenn sich bei ihr das Verhältnis zwischen Arbeitskraft und Boden verschiebt. Da in den Familienwirtschaften die Arbeitsmacht der Familie eine gegebene Grösse ist, muss sich hier die Betriebsfläche häufig mehren, wenn der Betrieb als Familienwirtschaft bestehen bleiben soll und wenn nicht die Möglichkeit besteht, durch weitere Intensivierung den Roh- 
ertrag auf der bestehenden Fläche erheblich zu steigern. Letzteres war zweifellos in vielen Betrieben der Fall, ein Zeichen dafür, dass sie bisher zu extensiv bewirtschaftet wurden. Wo weder die Möglichkeit zur Intensivierung noch die Möglichkeit zur Betriebsvergrösserung gegeben ist, werden die kleinen Familienwirtschaften dann zu Nebenerwerbswirtschaften. Wie bereits erwähnt, werden aber dafür im Zeitalter des steigenden Wohlstandes Gesindebetriebe zu reinen Familienbetrieben.

Zur Zeit ist die wirtschaftliche Lage der bäuerlichen Familienwirtschaften in den einzelnen Ländern stark abhängig von dem Stand der jeweiligen Volkswirtschaft und von der Aufgabe, die der Landwirtschaft schlechthin gestellt ist. Sollen und müssen in der Landwirtschaft möglichst viele Menschen Brot u n d Arbeit finden wie z.B. in Italien, oder besteht in der Volkswirtschaft Mangel an Arbeitskräften, wie z.B. in Westdeutschland, wo es Aufgabe der Landwirtschaft geworden ist, mit möglichst wenig Arbeitskräften ausreichende Mengen an Nahrungsmitteln zu produzieren. Die unterschiedlichen Meinungen zeigten sich sehr deutlich gelegentlich der Tagung der Gesellschaft für Agrarpolitik und Agrarsoziologie im Jahre 1957, wo der Vertreter Englands zum Ausdruck brachte, dass es dort das Ziel sei, mit 6 Arbeitskräften je 100 ha auszukommen, während ein Vertreter Italiens die Schaffung von Siedlungen mit 3-15 ha befürwortete, damit mindestens 30 Arbeitskräfte je 100 ha Arbeit fänden.

Dort, wo ein Uberschuss an Arbeitskraft besteht, wo die volkswirtschaftliche Arbeitsteilung noch nicht stark ausgeprägt ist, wo nicht genügend Arbeitsplätze vorhanden sind, um einen Arbeitskraftüberhang aufzunehmen, ist die Familienwirtschaft alter Prägung zu finden. Es handelt sich um Betriebe im Typ der Selbstversorgungswirtschaft, die nur einen geringen Teil ihrer Produkte dem Markt zuführen. Die relativ kleine Bodenfläche gibt der bäuerlichen Familie Brot und Arbeit. Man lebt genügsam und zufrieden, weil man die Wohlhabenheit der anderen nicht kennt. Sobald diese Länder oder Landesteile industriell oder sonstwie volkswirtschaftlich aufgeschlossen werden, ändern sich die Verhältnisse schnell. Der Familienbetrieb alter Prägung wird zur Nebenerwerbswirtschaft oder gibt die Landwirtschaft auf und der Boden wird von Nachbarbetrieben übernommen, die dann zu produktiven Familienbetrieben neuer Prägung werden.

Das Ziel des EWG-Vertrages ist es, die Volkswirtschaften der beteiligten Länder anzugleichen. Wenn EWG und EFTA zu einem grossen europäischen Wirtschaftsblock zusammenwachsen, so muss dann in diesem ebenfalls eine Nivellierung der Volkswirtschaften dieser Grossraumwirtschaft eintreten. Wie es heute in den einzelnen Ländern ärmere und reichere Gebietsteile gibt, so werden selbstverständlich auch in der Grossraumwirtschaft gewisse Unterschiede im Lebensund Ernährungsstandard bestehen.

Wenn man aber den Äusserungen der massgebenden EWG-Experten folgen darf, so dürfte der Lebens- und Ernährungsstandard in der Grossraumwirtschaft nicht unerheblich ansteigen. Der unterschiedliche Verzehr an animalischen Lebensmitteln lässt einen Schluss zu, welche Verbrauchsreserven gerade an den Produkten bestehen, die die bäuerliche Familienwirtschaft erzeugt. Je Kopf der Bevölkerung werden z.B. an Fleisch verzehrt in Frankreich rd. $80 \mathrm{~kg}$, in Deutschland rd. $50 \mathrm{~kg}$ und in Italien etwas über $20 \mathrm{~kg}$. Wird der Verbrauch in den beiden letztgenannten 
Ländern auf den französischen (und amerikanischen) Stand gehoben, so können die Erträge der Futterflächen noch gewaltig gesteigert werden. Es ist nämlich zu bedenken, dass die Kalorienverluste bei der Veredlung sehr beachtlich sind, sie betragen z.B. bei der Milchproduktion $70 \%$, bei der Kalbfleischerzeugung 95\%. Bei steigendem Verzehr an animalischen Nahrungsmitteln müssen sich daher die Bodenerträge mehr erhöhen als der Nahrungsverbrauch. Dem Gespenst der drohenden Überproduktion sollte man diese Tatsache gegenüberhalten. Aufgabe der Agrarpolitik wird es aber zum Schutze der Familienwirtschaften sein müssen dafür zu sorgen, dass die Futtermittelimporte und insbesondere die Importe an Ölfrüchten nicht zu gross werden.

Der sich aus der Statistik ergebende Entwicklungstrend wie auch ökonomische Überlegungen lassen den Schluss zu, dass dem reinen Familienbetrieb auch in Zukunft im europäischen Raum eine grosse Bedeutung zukommt, ja er wird an Bedeutung gewinnen. Nur muss man sich von der Vorstellung lösen, dass der Familienbetrieb an eine bestimmte Betriebsgrössenklasse gebunden ist. Die Dynamik der volkswirtschaftlichen und der agrartechnischen Entwicklung bringen es vielmehr mit sich, dass sich hier Wandlungen vollziehen, die mit politischen Mitteln höchstens gebremst, aber wohl kaum aufgehalten werden können. 